\title{
Mesenchymal Stem Cells Attenuate Acute Lung Injury in Mice Partly by Suppressing Alveolar Macrophage Activation in a PGE2-dependent Manner
}

\section{Gaojian Wang}

Zhejiang University School of Medicine Sir Run Run Shaw Hospital

\section{Yaping Zhang}

Zhejiang University School of Medicine Sir Run Run Shaw Hospital

Nianqiang $\mathrm{Hu}$

Zhejiang University School of Medicine Sir Run Run Shaw Hospital

Qinxue Liu

Zhejiang University School of Medicine Sir Run Run Shaw Hospital

\section{Fengjie Ma}

Zhejiang University School of Medicine Sir Run Run Shaw Hospital

Junran Xie ( $\nabla$ xiejunran@zju.edu.cn )

Shao Yifu Hospital: Zhejiang University School of Medicine Sir Run Run Shaw Hospital https://orcid.org/0000-0003-0853-8123

\section{Research}

Keywords: Mesenchymal stem cell, Alveolar macrophage, Acute lung injury, Prostaglandin E2, RNAsequence

Posted Date: July 7th, 2021

DOl: https://doi.org/10.21203/rs.3.rs-664786/v1

License: (a) This work is licensed under a Creative Commons Attribution 4.0 International License. Read Full License 


\section{Abstract}

Background: Mesenchymal stem cell have shown therapeutic effect on acute lung injury, MSC could be activated when added to inflammatory environment and in turn suppress inflammation, yet the mechanism is complex and not understood.

Methods: To determine the effect of MSC on ALI and alveolar macrophage activation, MSCs were administered to ALI mice and co-cultured with activated MH-S cells (alveolar macrophage cell line). To find the genes critical for MSC's immunosuppressive effects, rest and activated MSCs induced by inflammatory MH-S cells were harvested for RNA-seq. To prove that PGE2 participates in the immunosuppressive effects of MSC, COX2 inhibitor and PGE2 receptor antagonist were added to the coculture system and administrated to ALI mice.

Results: The intratracheal administration of MSCs attenuated ALI and suppressed alveolar macrophages activation in vivo, the activation of MH-S cells was also significantly reduced after co-culturing with MSCs in vitro. The RNA-seq data of rest and activated MSCs suggested that the Ptgs2 gene may play an important role in MSC exerting immunosuppressive effects. Correspondingly, we found that the COX2 protein and PGE2 released by activated MSCs were increased dramatically after co-culturing with MH-S. The use of COX2 inhibitor NS-398 restrained the secretion of PGE2 and reversed the suppressive effect on macrophages activation of MSCs in vitro. Furthermore, GW627368X, a selective antagonist of PGE2 receptor (EP4 receptor), also reversed the inhibitory effects of MSCs on alveolar macrophages and their protective effects on ALI mice.

Conclusions: MSC attenuate ALI partly through suppressing alveolar macrophage activation via PGE2 binding to EP4 receptor.

\section{Background}

Acute lung injury (ALI) and its clinical form, acute respiratory distress syndrome (ARDS), are characterized by the abrupt occurrence of significant hypoxemia accompanied by diffuse pulmonary infiltration, manifesting as excessive pulmonary and even systemic inflammation [1, 2]. Despite the progress made in supportive therapy and pharmaceutical research, such as extracorporeal membrane oxygenation (ECMO) and updated guidance on appropriate drug interventions, the morbidity of ALI/ARDS remains high [3].

Alveolar macrophages (AMs) are the predominant immune cells in the alveoli and act as the first line of defense of innate immunity there $[4,5]$. Like other tissue macrophages, AMs are considered to maintain homeostasis by clearing endogenous waste and invading exogenous pathogens. AMs can also be activated, secreting proinflammatory cytokines that recruit neutrophils and other immune cells $[6,7]$. However, in ALI, the excessive activation of AMs triggers uncontrollable inflammatory cascade, causing tissue damage. Given their strong ability to suppress immune cells activation, stem cells may provide an effective way to resolve ALI $[8,9]$. 
Mesenchymal stem cells (MSCs) are multipotent stem cells derived from different tissues, including bone marrow, umbilical cord, and placenta. A growing number of studies had identified that MSCs can exert immunosuppressive effect [10-12], in addition, reports also had pointed out that rest MSCs do not inherently own this power $[13,14]$, which can be gained by inflammatory cytokines stimulation and reversely suppressed immune cells activation, forming a negative feedback. For example, in ALI model, the engrafting MSCs were activated by strong inflammatory stimuli in lungs and acquired immunosuppressive capacity. Hence, analysis of gene expression alteration of rest and activated MSCs may shed light on their immunosuppressive mechanisms.

Based on the above background, we hypothesized that MSCs attenuate ALI by suppressing AM activation and attempted to explore the potential immunosuppressive mechanisms of MSCs by bulk RNA-seq.

\section{Methods}

\section{Mouse Bone Marrow-Derived Mesenchymal Stem Cells (MSCs) and MH-S Cells}

Mouse bone marrow-derived mesenchymal stem cells (MSCs) were purchased from Cyagen Biosciences (Guangzhou, China) and identified by cytometry (Fig.1A). The passage number of MSCs used in the experiment was under 15. MSCs were cultured in DMEM/F12 medium (Meilunbio, Dalian, China) with 8\% fetal bovine serum (Cellmax, Beijing, China) and 1\% penicillin/streptomycin (Meilunbio). MH-S cells were obtained from Otwo Biotech (Shenzhen, China) and cultured in DMEM medium (Gibco, Masschusets, USA) with $8 \%$ fetal bovine serum and $1 \%$ penicillin/streptomycin. All cells grew in a humidified $5 \% \mathrm{CO}_{2}$ sterile incubator at $37^{\circ} \mathrm{C}$.

\section{In Vivo ALI Mouse Model}

Male C57BL/ 6 mice, 8-10 weeks of age, were purchased from Zhejiang Academy of Medical Sciences (Hangzhou, China). The mice were kept in Sir Run Run Shaw Hospital (Hangzhou, China) with a 12-h light/dark cycle and free access to food and water. The mice were anesthetized and instilled with Lipopolysaccharide (LPS, $5 \mathrm{mg} / \mathrm{kg}$ for most experiments or $10 \mathrm{mg} / \mathrm{kg}$ for the survival experiment) (Escherichia coli, 0111:B4; Sigma-Aldrich, USA) in $50 \mathrm{ml}$ of PBS through a 24G catheter inserted into the trachea, establishing the ALI model. Control mice were instilled with $50 \mathrm{ml}$ of PBS in the same way. All experiments were performed in conformity to Institutional Animal Care and Use Committee-approved protocols.

\section{Experimental Design}

The experimental protocol is shown in Fig. 1B. The mice were divided into three groups: PBS+PBS group, LPS+PBS group, and LPS+MSC group. MSCs $\left(2 \times 10^{6}\right.$ cells per mouse) in $50 \mathrm{ml}$ of PBS or PBS alone were instilled intratracheally (IT) at $4 \mathrm{~h}$ after LPS instillation in the LPS+MSC group and the other groups, respectively. The mice were euthanized $48 \mathrm{~h}$ after ALI model establishment for further experiments or observed for 2 weeks to record their body weight and survival rate 


\section{Mouse in Vivo imaging}

Luc-PGK-Puro lentivirus was transfected to MSCs following the manufacture's instructions, the stable luciferase expression MSCs were obtained after puromycin filtration (named $\mathrm{Luc}^{+} \mathrm{MSC}$ ). The mice were divided into PBS instilled group (control group) and LPS instilled group (ALI model group). Luc ${ }^{+} \mathrm{MSC}$ ( $2 \times 10^{6}$ cells per mouse) in $50 \mathrm{ml}$ PBS was instilled intratracheally (IT) at $4 \mathrm{~h}$ after model establishment. D-luciferin $(15 \mathrm{mg} / \mathrm{ml})$ was injected intraperitoneally $(10 \mathrm{ul} / \mathrm{g})$ at $2 \mathrm{~h}, 24 \mathrm{~h}$ and $48 \mathrm{~h}$ after Luc ${ }^{+} \mathrm{MSC}$ instillation. Fluorescence signal was detected at $30 \mathrm{~min}$ after D-luciferin injection with imaging system (PerkinElmer, Caliper IVIS Lumina II, USA).

\section{Arterial Oxygenation Analysis}

For arterial oxygenation analysis, mice were first anesthetized and the carotid artery was adequately exposed. The distal end was ligatured with silk while the proximal end was occluded. A PE-10 catheter was then inserted through a $V$ notch and arterial blood was collected using a $1 \mathrm{ml}$ heparinized syringe through the catheter after removing the occlusion. The mice were then euthanized for other experiments. A blood gas analyzer was used to determine arterial oxygenation.

\section{Bronchoalveolar Lavage Fluid}

For the collection of bronchoalveolar lavage fluid (BALF), mice were euthanized, after which the trachea was exposed quickly and then cannulated using a $24 \mathrm{G}$ catheter. This catheter was fixed using silk ligature. A total of $0.8 \mathrm{ml}$ of cold PBS-EDTA solution ( $2 \mathrm{mM}$ ) was infused, which was maintained for 1 min to collect BALF. This was repeated five times and the collected BALF was temporarily stored on ice.

\section{Pulmonary Capillary Permeability Measurement}

For measurement of the permeability of pulmonary capillaries, the right upper lobe of the lungs was removed and its wet weight was recorded. Then, its dry weight was measured after placing the lungs in a $70^{\circ} \mathrm{C}$ incubator for $24 \mathrm{~h}$. The wet/dry weight ratio was then calculated. The collected BALF was filtrated with a $40 \mu \mathrm{m}$ strainer and then centrifuged at $300 \mathrm{~g}$ for $5 \mathrm{~min}$. The resulting supernatant was collected, the protein content of which was measured by BCA assay.

\section{Histology}

For histological analysis, the right lobe of the lungs was excised, fixed with $10 \%$ formalin for $24 \mathrm{~h}$, embedded in paraffin, cut into $5 \mu \mathrm{m}$ slices, and finally stained with hematoxylin and eosin.

\section{Flow Cytometry}

For flow cytometry, the centrifuged cells in BALF were suspended in PBS, incubated for 10 min with FcR blocking reagents, and then incubated for $15 \mathrm{~min}$ with APC-CY7 fixable viability dye, followed by another 20 min with PE-F4/80, FITC-CD11b, and PE-CY7-CD86 antibodies (Biolegend, USA). After incubation, the 
cells were washed with PBS twice, after which R-PE IMag particles (BD Pharmingen, USA) were added and incubated for $30 \mathrm{~min}$ on ice, followed by enrichment of the PE-labeled cells by magnetic cell sorting with a magnetic frame. The remaining cells were then washed twice and fix/perm treatment was performed, after which the cells were incubated with APC-CD206 (Biolegend, USA) antibody for 20 min. The stained cells were washed and suspended in $300 \mathrm{ml}$ of buffer for flow cytometry (BD LSRFortessa, USA). The cultured MH-S cells were incubated with FcR blocking reagents, APC-CY7 fixable viability dye, FITC-CD11b, and PE-CY7-CD86 antibodies for flow cytometry. The results were analyzed by FlowJo software.

\section{Co-culture of MSCs and MH-S Cells}

For the co-culture of MSCs and MH-S cells, a six-well transwell co-culture system $(0.4 \mu \mathrm{m}$ pores; Corning, USA) was applied. MSCs $\left(2 \times 10^{5}\right.$ cells/well) were seeded in the six-well plates and MH-S cells $\left(5 \times 10^{5}\right.$ cells/well) were grown in the upper wells of the six-well transwell co-culture system overnight. Then, the upper wells were transferred to the lower wells that contained MSCs, establishing a co-culture system, and the medium was changed to DMEM/F12 complete medium with LPS (200 ng/ml) and IFN-y (20 $\mathrm{ng} / \mathrm{ml})$, which were used for activating MH-S cells. NS-398 (10 $\mu \mathrm{M}$; APExBIO, USA) or GW627368X (10 $\mu \mathrm{M}$; MCE MedChemExpress, China) was added to the medium as needed. The cells were harvested after $24 \mathrm{~h}$ for subsequent experiments.

\section{Western Blotting}

For western blotting, the cells were lysed in cell lysis buffer (Beyotime, China) with protease inhibitor. Proteins were separated by SDS-PAGE and transferred to PVDF membranes, after which the membranes were blocked with $5 \%$ nonfat milk in TBST for $1 \mathrm{~h}$ at room temperature. These membranes were then incubated with the primary antibodies overnight at $4^{\circ} \mathrm{C}$ on a rotator. Membranes were then incubated with HRP-conjugated secondary antibody (Beyotime) at room temperature for $2 \mathrm{~h}$, enhanced chemiluminescence reagents were added to visual bands, and the grayscale value was quantified using ImageJ software. The primary antibodies used were for iNOS, GAPDH (CST, USA), and COX2 (Bioworld, China).

\section{Quantitative Real-time PCR (qPCR)}

For quantitative real-time PCR, total RNA was isolated from cells using a Total RNA Isolation Kit (Vazyme, China) and measured for optical density at $260 \mathrm{~nm}$. Subsequently, total RNA was reverse-transcribed to cDNA with a reverse transcription kit (Vazyme). qPCR was carried out with a two-stage program. Primers used for qPCR were for mouse TNF-a, Ccl2, Ccl3, Ccl5, Cxcl2, and GAPDH (Tsingke Biotechnology, China). The sequences were as follows: mouse TNF-a: 5'-CCCTCACACTCAGATCATCTTCT-3'(F), 5'GCTACGACGTGGGCTACAG-3'(R), CCl2: 5'-TTAAAAACCTGGATCGGAACCAA-3'(F), 5'-

GCATTAGCTTCAGATTTACGGGT-3'(R), CC/3: 5'-TTCTCTGTACCATGACACTCTGC-3'(F), 5'CGTGGAATCTTCCGGCTGTAG-3'(R), CCI5: 5'-GCTGCTTTGCCTACCTCTCC-3'(F), 5'TCGAGTGACAAACACGACTGC-3'(R), CXCl2:5'- CCAACCACCAGGCTACAGG-3'(F), 5'- 
GCGTCACACTCAAGCTCTG-3'(R), and GAPDH: 5'-AGGTCGGTGTGAACGGATTTG-3'(F), 5'-

TGTAGACCATGTAGTTGAGGTCA-3'(R).

\section{Enzyme-Linked Immunosorbent Assay (ELISA)}

The concentrations of TNF- $a$, Ccl2 and PGE2 factors were measured using ELISA with mouse TNF-a and Ccl2 ELISA kits (Elabscience, China) and PGE2 ELISA kits (R\&D, USA), following the manufacturers' instructions.

\section{Bulk RNA-seq and Data Analysis}

Rest MSCs before co-culture and activated MSCs after co-culture were collected (Fig. 5A), after which their total RNA was extracted, quantified, and qualified. RNA sequencing was performed with an Illumina platform. Raw reads were first processed using in-house Perl scripts. Then, clean reads were obtained by removing reads containing an adapter, reads containing a poly $(\mathrm{N})$ region, and low-quality reads from the raw reads. A reference genome and gene model annotation files were directly downloaded from a genome website. The reference genome index was built and paired-end clean reads were aligned to the reference genome by Hisat2 v2.0.5. Then, featureCounts v1.5.0-p3 was applied to count the reads mapped to each gene. Analysis of differentially expressed genes (DEGs) between two groups was performed using the DESeq2 R package (1.16.1). Genes with an adjusted $p$-value $<0.05$ found by DESeq2 were considered to be differentially expressed. A cluster heatmap of the DEGs was created using the ComplexHeatmap R package (2.6.2). Gene Ontology (GO) and Kyoto Encyclopedia of Genes and Genomes (KEGG) pathway analyses were performed with the web-based Gene SeT AnaLysis Toolkit (http://www.webgestalt.org). To construct a transcription factor (TF)-target gene (TG) network, we collected mouse TF-TG interactions from TRED and KEGG databases, resulting in a list of TF-TG pairs (Supplemental S1). Screening was performed to identify the target genes and transcription factors among the DEGs and the network was visualized using Cytoscape software.

\section{Statistical Analysis}

All statistical analyses were performed using SPSS 23.0 software and the data were visualized using GraphPad Prism 7.0. Kaplan-Meier test was applied for survival curve comparison. Comparisons between multiple groups were performed using one-way analysis of variance (ANOVA). Comparisons between two groups were performed using $T$ test. The data are expressed as the mean \pm SD and a value of $p<0.05$ was considered significant.

\section{Results}

\section{Identification of MSCs and their Ability to Attenuate ALI}

MSCs were identified by flow cytometry. As expected, these cells expressed the MSC markers CD29, CD44, Sca-1, and CD34, but not CD117 (Fig. 1A). The mouse in vivo imaging suggested that MSCs engrafted to 
both lungs and resided more than $48 \mathrm{~h}$ in ALI mouse, while instilled Luc ${ }^{+}$MSCs in control mouse were almost undetected after $24 \mathrm{~h}$ and entirely disappeared after 48h (Fig. 1C), these indicated MSCs could be attracted to inflammation.

The survival rate of ALI mice treated with MSCs was significantly higher than that of mice treated with PBS alone (Fig. 2A). After LPS instillation, MSC treatment reversed the weight reduction on the 5th day, while mice in the LPS + PBS group continued losing weight until the 11th day; on the 14th day, the weight in this latter group was significantly lower than that in the LPS + MSC group (Fig. 2B). Similarly, the P/F ration in the LPS + PBS group declined, while that in the LPS + MSC group was significantly higher, approaching the normal value in the PBS + PBS group (Fig. 2C). MSC treatment also altered lung permeability, with the total protein in BALF and the lung wet/dry ratio being clearly improved in the LPS + MSC group (Fig. 2D, 2E). The TNF-a concentration in BALF also showed a statistically significant difference between LPS + MSC group and LPS + PBS group (Fig. 2F). The extent of pulmonary pathological injury was determined by H\&E staining. The lungs in the LPS + PBS group exhibited the greatest damage, alveolar epithelium cells were widely broken, resulting extensive collapse alveolar and invalid cavity, while the profile of alveoli in LPS + MSC group was still clear with less collapse alveolar (Fig. 3A). Taken together, these results indicate that MSCs effectively attenuated the LPS-induced ALI.

\section{MSCs Suppress AM Activation In Vivo and In Vitro}

According to the designed gate strategy in Fig. 3B, the AM cluster was isolated by the presence of F4/80 and CD206 double positivity (Fig. 3B). CD11b and the costimulatory molecule CD86, which were expressed at low levels on the rest AM surface, exhibited increases in expression after LPS treatment (Fig. $2 \mathrm{E})$, suggesting the activation of AMs and their adoption of a proinflammatory state. The MSC treatment significantly suppressed this AM activation. As further research, cells of the mouse AM cell line MH-S were used for an in vitro experiment. Co-culture of MSCs and activated MH-S cells (activated by LPS plus IFN- $\gamma$ ) led to the inhibition of macrophage activation, manifesting as dramatic declines in the mRNA and protein levels of TNF- $a$ and chemokines (Fig. 4A, 4B), decreased inducible nitric oxide (iNOS) protein (Fig. 4C), and reduced CD11b and CD86 expression on the cell surface (Fig. 4D). These findings suggested the inhibition of AM activation by MSCs.

\section{MSC RNA-seq Data Analysis}

Rest MSCs before co-culture and activated MSCs after co-culture were harvested and total RNA was extracted for RNA-seq (Fig. 5A). As shown in Fig. 5B, 2754 DEGs (differential expression genes) were upregulated and 2514 DEGs were downregulated of activated MSCs compared with the levels in the rest MSCs. All DEGs were classified into four clusters according to the gene expression pattern (Fig. $5 \mathrm{C}$ ). To further investigate the potential roles of upregulated genes in clusters 1 and 4, GO enrichment and KEGG pathway analyses were implemented. The top $10 \mathrm{GO}$ terms and pathways shown in Fig. 5D and 5E were significantly enriched $(p<0.001)$. The enriched terms and pathways in cluster 1 , such as response to cytokines, amide biosynthetic process, ribosome biogenesis, and TNF/IL-17/NOD-like signaling pathway, 
indicated the identified genes function in inflammatory response and protein metabolism. In addition, the analysis of cluster 4 strongly indicated the genes function in increasing protein metabolism. These results indicated the effects of activated MSCs in enhancing metabolism and reactions to inflammatory stimuli.

To explore the intracellular signaling, target genes of upregulated DEGs were ranked by the total number of upstream transcription factors. The top 10 target genes and the molecules acting upstream of them were used to construct a network (Fig. 5F). Ptgs2, Nos2, Hmox1, and Tnfaip3 are generally considered to be genes related to immunosuppression; among these, Ptgs 2 was modulated by the most transcription factors, meaning that it was more finely regulated and participated more widely in the immunosuppressive process. Hence, the Ptgs2 gene was chosen for further study.

\section{PGE2 Secreted by MSCs Contributes to Suppressing AM Activation}

The Ptgs 2 gene encodes the COX2 protein, which is the key enzyme in PGE2 synthesis. The expression of COX2 protein in MSCs was shown to be time-dependently induced after co-cultured with activated macrophages (Fig. 6A); at the same time, the level of PGE2 in both cell supernatants and BALF dramatically increased after adding MSCs (Fig. 6B, 6C). To clarify the role of PGE2 in regulating macrophage activation, the COX2 inhibitor NS-398 was used. The amount of PGE2 secreted by MSCs decreased significantly in the presence of this inhibitor (Fig. 6B). Meanwhile, NS-398 eliminated the suppressive effect of MSCs on TNF-a release (Fig. 6D) and the CD86 expression of MH-S cells (Fig. 6E). The above results demonstrated that activated MSCs secreted a large amount of PGE2, which contributed to the suppression of AM activation.

\section{MSCs- PGE2- E-Prostanoid Receptor 4 Axis Participate in Suppressing AM Activation and Relieving ALI}

There are four PGE2 receptors, E-Prostanoid receptors 1-4 (EP1-4), on the surface of AM. Previous studies indicated that PGE2 exerts its anti-inflammatory effects through EP4. To further characterize the role of PGE2 in the suppression of AM activation, a selective EP4 antagonist, GW627368X, was applied. In an in vitro experiment, GW627368X significantly reversed the inhibitory effect of MSCs on the TNF-a secretion of MH-S cells (Fig. 7A). In an in vivo experiment, GW627368X was IT administered along with MSCs, which significantly reversed the protective effect of MSCs on the survival rate (Fig. 7B), and the suppression of TNF-a release (Fig. 7C). These findings revealed that the inhibitory effects of MSCs were facilitated through the binding of PGE2 to the EP4 receptor on AMs.

\section{Discussion}

ALI is a serious inflammatory disease, characterized by uncontrollable, excessive inflammatory damage to the alveolar epithelium [1]. We investigated the effect of MSCs on ALI and explored the immunosuppressive mechanisms of MSCs by RNA-sEq. In our experiment, we drew the following conclusions from findings upon the IT administration of MSCs in vivo or co-culture with alveolar macrophages in vitro: (1) MSCs alleviated ALI, (2) MSCs suppressed AMs activation both in vivo and in 
vitro, and (3) MSCs facilitated immunosuppression by secreting PGE2, which bound to EP4 receptor. Finally, the evidence suggested that MSCs attenuate ALI by suppressing the activation of AMs in a PGE2dependent manner.

The beneficial effects of MSCs on activated macrophages have been described in many studies, such as regulating Kupffer cell polarization in acute liver injury [12,16], inhibiting intestine-resident macrophages inflammation in colitis [11, 17], and suppressing microglial activation in the nervous system [18, 19]. However, to date, there has been little research on MSCs acting on alveolar macrophages in ALI [10, 2022].

In our studies, MSCs clearly attenuated ALI and inhibited alveolar macrophage activation both in vivo and in vitro. Macrophage activation is well known to include proinflammatory M1 activation and antiinflammatory M2 activation [15]. However, our research showed the significant suppression of M1-like activation of AMs after treatment with MSCs, while there was no meaningful change toward M2-like polarization, described as increased secretion of anti-inflammatory cytokines (IL-10, TGF- $\beta$ ) and high expression of CD206 and Arg-1. We attempted to explain this situation, firstly, the polarization of macrophages varies with the inflammatory process. They are activated to the M1 type at the beginning of inflammation, helping to build an inflammatory microenvironment, later or accepting new stimuli, they may switch to M2 type to facilitate tissue repair [20,23]. This conversion may take several days, which varies depending on the severity and localization of inflammation. MSC treatment can accelerate this process. In our research, we euthanized ALI mice $48 \mathrm{~h}$ after LPS instillation, it may be too early to observe the M2 activation. Secondly, the familiar M1/M2 classification is not absolutely definitive and arguments about its validity have regularly been reported since it was first suggested [24, 25]. Owing to macrophage's plasticity, different tissue-resident macrophage presents variable morphology, function, and response; as such, it is not appropriate to apply a single, simple classification system to all macrophages. Alveolar macrophages originate from fetal liver monocytes or are quickly supplemented by hematopoietic stem cells when exhausted. They are long-lived and self-sustaining macrophages populating in alveoli, which are characterized as $\mathrm{CD} 45^{\mathrm{hi}}, \mathrm{CD} 11 \mathrm{c}^{+}, \mathrm{CD}_{11 \mathrm{~b}^{-}}$, SiglecF ${ }^{+}, \mathrm{CD} 206^{+}$, and F4/80 int $[6,26]$. Unlike spleen macrophages or peritoneal macrophages, CD206 is constantly expressed on AMs, rather than being a symbol of M2 polarization. Meanwhile, AMs exhibit high expression of CD200R, which can negatively regulate the inflammatory response by binding to the ligand CD200L on the epithelium [27]. These findings indicate that AMs may exhibit different anti-inflammatory phenotypes after MSC treatment, rather than the well-known M2 type, the detailed mechanisms of this require further study.

Similar to macrophages, MSCs also possess high plasticity[28], the immunosuppressive ability of MSCs is induced by inflammatory mediators rather than being inherent. This was proven by the finding that MSCs pretreated by exposure to inflammatory conditions had a better anti-inflammatory effect [29, 30]. In ALI mouse model, instilled LPS and inflammatory cytokines secreted from damaged epithelium firstly activated alveolar macrophage, then activated AMs worked as a trigger evoking more damage, forming a vicious circle, when rest MSCs engrafted to lungs, they would be soon activated by inflammatory stimuli 
from immune cells or injured tissues and suppressed excessive AM activation by secreting antiinflammatory cytokines (Fig. 8). To figure out the anti-inflammatory cytokines from activated MSCs, we performed bulk RNA-seq of rest and activated MSCs. Known from RNA-seq data, 2754 upregulated genes of activated MSCs were classified into two clusters according to the gene expression pattern. Based on GO enrichment and KEGG pathway analyses, functional annotation indicated genes in cluster 1 involved in inflammatory response and protein metabolism, and genes in cluster 4 involved in protein metabolism. The gene expression alteration suggested that activated MSCs respond to inflammation and enhance metabolism. According to the transcription factor-target gene network, the included target genes are under complex regulation by upstream transcription factors, suggesting that these genes play critical roles in MSCs inflammation response. The top 10 target genes in network could be divided into five categories according to gene function: (1) angiogenesis-related gene (Vegfa) [31, 32]; (2) proinflammatory genes ( $C \mathrm{Cl} 2, \mathrm{CxCl} 10$, and II-6); (3) immunosuppression-related genes (Ptgs2, Nos2, Hmox 1, and Tnfaip3); (4) coagulation-related gene (Serpine1); and (5) cell cycle and metabolism-related genes (Cdkn1a, Mt1, and Mdm2). This list of genes provides an overview, albeit a somewhat limited one, of the whole signaling network of MSCs. As we had discovered in network, activated MSCs could secrete both proinflammatory and anti-inflammatory cytokines, accumulating evidence had indicated the bilateral immunomodulatory capacity of MSCs, the direction of their effects was largely affected by the inflammatory context. For example, in early inflammatory stage or chronic inflammation, without sufficient stimuli, MSCs release proinflammatory cytokines, such as chemokines and IL-6, to induce immune response. On the other hand, MSCs could be activated immediately by intense inflammation and then release anti-inflammatory bioactive substances [33, 34].

According to network, Ptgs2 may play important role in MSC's anti-inflammatory capacity. Our experiment also proved that the expression of COX2 of MSCs was induced by inflammation, leading to the release of an enormous amount of PGE2. The use of NS-398 confirmed the precise suppressive role of PGE2 on macrophage activation, just as the RNA-seq data had suggested. The interaction between PGE2 and macrophages is established through EP1-4 receptors on the surface of macrophages. Previous studies demonstrated that the anti-inflammatory effects of PGE2 are mediated through the EP4 receptor [35]. Application of the selective EP4 antagonist GW627368X significantly reversed the immunosuppressive effects of MSCs both in vivo and in vitro, which further confirmed the important role of the MSC-PGE2EP4 axis in restraining alveolar macrophage activation and relieving ALI. The MSCs and immune cells interaction network is complex, macrophage bulk RNA-seq and alveolar immune cells scRNA-seq will be further completed to explain the immunosuppressive mechanisms of MSCs in a more comprehensive way.

This study has some limitations. First, the long-term effect of MSCs on alveolar macrophage activation in ALI remains unclear. Longer observation or repeated administration of MSCs may promote the M2 activation of alveolar macrophages and enhance the clinical significance of MSC therapy for ALI. Second, the immune cells in ALI mice comprised several types, including alveolar macrophages, neutrophils, lymphocytes, and recruited monocytes, all of which influence ALI development. However, we cannot explain the immunosuppressive effects of MSCs on all of these cell types. Finally, the 
mechanisms by which PGE2 acts to suppress the activation of alveolar macrophages and other possible mechanisms concerning the immunosuppressive properties of MSCs need further study.

\section{Conclusion}

In conclusion, the administration of MSCs was shown to attenuate ALI, partly by suppressing the activation of alveolar macrophages by increasing the synthesis of PGE2, which binds to the EP4 receptor on these macrophages. The results of our study demonstrated that MSC therapy may provide more effective therapeutic options for acute lung injury and ARDS.

\section{Abbreviations}

MSCs Mesenchymal stem cells

Luc+ MSC Luciferase expression MSCs

AMs Alveolar macrophages

ALI Acute lung injury

TF Transcription factor

TG Target gene

EP4 E-Prostanoid Receptor 4

\section{Declarations}

\section{Ethics approval and consent to participate}

All experiments were performed in conformity to Institutional Animal Care and Use Committee-approved protocols.

\section{Consent for publication}

Not applicable.

\section{Available of data and materials}

The data that support the findings of this study can be obtained from the corresponding author upon reasonable request.

\section{Competing interests}

The authors declare that they have no competing interests. 


\section{Funding}

This work was supported by grants from the National Natural Science Foundation of China (No. 8170080655).

\section{Author's contributions}

Gj.W contributed to experiment design, performed all experiments and most data analysis, and wrote manuscript. Yp.Z, Nq.H and Jr.X contributed experiment design, administrative support and financial support. Qx.L and Fj.M performed partial data analysis. All authors provided supervision and approved the final manuscripts.

\section{Acknowledgements}

Not applicable.

\section{References}

1. A.P. Wheeler, G.R. Bernard, Acute lung injury and the acute respiratory distress syndrome: a clinical review, The Lancet, 2007369 1553-64.

2. B. Xu, S.S. Chen, M.Z. Liu, C.X. Gan, J.Q. Li, G.H. Guo, Stem cell derived exosomes-based therapy for acute lung injury and acute respiratory distress syndrome: A novel therapeutic strategy, Life Sci, 2020 254117766.

3. Y. Zheng, J. Liu, P. Chen, L. Lin, Y. Luo, X. Ma, et al., Exosomal miR-22-3p from human umbilical cord blood-derived mesenchymal stem cells protects against lipopolysaccharid-induced acute lung injury, Life Sci, 2021269119004.

4. B. Allard, A. Panariti, J.G. Martin, Alveolar Macrophages in the Resolution of Inflammation, Tissue Repair, and Tolerance to Infection, Front Immunol, 201891777.

5. T. Hussell, T.J. Bell, Alveolar macrophages: plasticity in a tissue-specific context, Nat Rev Immunol, 201414 81-93.

6. A.S. Neupane, M. Willson, A.K. Chojnacki, E.S.C.F. Vargas, C. Morehouse, A. Carestia, et al., Patrolling Alveolar Macrophages Conceal Bacteria from the Immune System to Maintain Homeostasis, Cell, 2020183 110-25 e11.

7. L.K. Johnston, C.R. Rims, S.E. Gill, J.K. McGuire, A.M. Manicone, Pulmonary macrophage subpopulations in the induction and resolution of acute lung injury, Am J Respir Cell Mol Biol, 2012 47 417-26.

8. N.J. Thomas, D. Spear, E. Wasserman, S. Pon, B. Markovitz, A.R. Singh, et al., CALIPSO: A Randomized Controlled Trial of Calfactant for Acute Lung Injury in Pediatric Stem Cell and Oncology Patients, Biol Blood Marrow Transplant, 201824 2479-86. 
9. J. Sun, X. Ding, S. Liu, X. Duan, H. Liang, T. Sun, Adipose-derived mesenchymal stem cells attenuate acute lung injury and improve the gut microbiota in septic rats, Stem Cell Res Ther, 202011384.

10. H. Deng, L. Wu, M. Liu, L. Zhu, Y. Chen, H. Zhou, et al., Bone Marrow Mesenchymal Stem Cell-Derived Exosomes Attenuate LPS-Induced ARDS by Modulating Macrophage Polarization Through Inhibiting Glycolysis in Macrophages, Shock, 202054 828-43.

11. H.J. Park, J. Kim, F.T. Saima, K.J. Rhee, S. Hwang, M.Y. Kim, et al., Adipose-derived stem cells ameliorate colitis by suppression of inflammasome formation and regulation of M1-macrophage population through prostaglandin E2, Biochem Biophys Res Commun, 2018498 988-95.

12. J. Wang, Y. Liu, H. Ding, X. Shi, H. Ren, Mesenchymal stem cell-secreted prostaglandin E2 ameliorates acute liver failure via attenuation of cell death and regulation of macrophage polarization, Stem Cell Res Ther, 20211215.

13. B. Wang, Y. Lin, Y. Hu, W. Shan, S. Liu, Y. Xu, et al., mTOR inhibition improves the immunomodulatory properties of human bone marrow mesenchymal stem cells by inducing COX-2 and PGE2, Stem Cell Res Ther, 20178292.

14. Y. Wang, X. Chen, W. Cao, Y. Shi, Plasticity of mesenchymal stem cells in immunomodulation: pathological and therapeutic implications, Nature Immunology, 201415 1009-16.

15. S. Gordon, F.O. Martinez, Alternative activation of macrophages: mechanism and functions, Immunity, 201032 593-604.

16. Y. Liu, H. Ren, J. Wang, F. Yang, J. Li, Y. Zhou, et al., Prostaglandin E2 secreted by mesenchymal stem cells protects against acute liver failure via enhancing hepatocyte proliferation, FASEB J, 201933 2514-25.

17. J.H. An, W.J. Song, Q. Li, S.M. Kim, J.I. Yang, M.O. Ryu, et al., Prostaglandin E2 secreted from feline adipose tissue-derived mesenchymal stem cells alleviate DSS-induced colitis by increasing regulatory T cells in mice, BMC Vet Res, 201814354.

18. M.A.A. Harrison, R.M. Wise, B.P. Benjamin, E.M. Hochreiner, O.A. Mohiuddin, B.A. Bunnell, AdiposeDerived Stem Cells from Obese Donors Polarize Macrophages and Microglia toward a ProInflammatory Phenotype, Cells, 202110.

19. Z. Zhang, X. Zou, R. Zhang, Y. Xie, Z. Feng, F. Li, et al., Human umbilical cord mesenchymal stem cellderived exosomal miR-146a-5p reduces microglial-mediated neuroinflammation via suppression of the IRAK1/TRAF6 signaling pathway after ischemic stroke, Aging-Us, 202113 3060-79.

20. A. Al-Rubaie, A.F. Wise, F. Sozo, R. De Matteo, C.S. Samuel, R. Harding, et al., The therapeutic effect of mesenchymal stem cells on pulmonary myeloid cells following neonatal hyperoxic lung injury in mice, Respir Res, 201819114.

21. H.X. Zhu, J.L. Gao, M.M. Zhao, R. Li, Y.X. Tian, X. Wang, et al., Effects of bone marrow-derived mesenchymal stem cells on the autophagic activity of alveolar macrophages in a rat model of silicosis, Exp Ther Med, 201611 2577-82.

22. D. Li, C. Wang, C. Chi, Y. Wang, J. Zhao, J. Fang, et al., Bone Marrow Mesenchymal Stem Cells Inhibit Lipopolysaccharide-Induced Inflammatory Reactions in Macrophages and Endothelial Cells, 
Mediators Inflamm, 201620162631439.

23. J. Yap, H.A. Cabrera-Fuentes, J. Irei, D.J. Hausenloy, W.A. Boisvert, Role of Macrophages in Cardioprotection, International Journal of Molecular Sciences, 201920.

24. P.J. Murray, Macrophage Polarization, in: D. Julius (Ed.) Annual Review of Physiology, Vol 79, 2017, pp. 541-66.

25. L. Cakarova, L.M. Marsh, J. Wilhelm, K. Mayer, F. Grimminger, W. Seeger, et al., Macrophage Tumor Necrosis Factor-alpha Induces Epithelial Expression of Granulocyte-Macrophage Colony-stimulating Factor Impact on Alveolar Epithelial Repair, American Journal of Respiratory and Critical Care Medicine, 2009180 521-32.

26. F. Ginhoux, M. Guilliams, Tissue-Resident Macrophage Ontogeny and Homeostasis, Immunity, 2016 44 439-49.

27. R. Mihrshahi, A.N. Barclay, M.H. Brown, Essential Roles for Dok2 and RasGAP in CD200 ReceptorMediated Regulation of Human Myeloid Cells, Journal of Immunology, 2009183 4879-86.

28. Q. Huang, X. Wei, Plasticity of mesenchymal stem cells in immunomodulation, SCIENTIA SINICA Vitae, 201646 799-808.

29. H. Zhen, J. Wang, L. Xue, J. Rui, X. Liu, Y. Chen, LPS-pretreated bone marrow stem cells as potential treatment for myocardial infraction, Frontiers in Bioscience-Landmark, 201217 1294-303.

30. P. Li, J. Liu, J. Sun, H. Duan, The LPS-Pretreated MSCs Supply a Positive Microenvironment for Tumor Cell Proliferation and Clone Formation, Current Protein \& Peptide Science, 2015 16 295-300.

31. Y. Sun, X. Xiong, X. Wang, The miR-590-3p/VEGFA axis modulates secretion of VEGFA from adiposederived stem cells, which acts as a paracrine regulator of human dermal microvascular endothelial cell angiogenesis, Human Cell, 202033 479-89.

32. Z. Tang, X. Wu, L. Hue, Y. Xiao, J. Tang, S. Zuo, et al., Circ-100290 Positively Regulates Angiogenesis Induced by Conditioned Medium of Human Amnion-Derived Mesenchymal Stem Cells Through miR449a/eNOS and miR-449a/VEGFA Axes, International Journal of Biological Sciences, 2020162131 44.

33. E. Kolaczkowska, P. Kubes, Neutrophil recruitment and function in health and inflammation, Nature Reviews Immunology, 201313 159-75.

34. N. Kim, S.-G. Cho, Overcoming immunoregulatory plasticity of mesenchymal stem cells for accelerated clinical applications, International Journal of Hematology, 2016 103 129-37.

35. J. Liu, A. Kuwabara, Y. Kamio, S. Hu, J. Park, T. Hashimoto, et al., Human Mesenchymal Stem CellDerived Microvesicles Prevent the Rupture of Intracranial Aneurysm in Part by Suppression of Mast Cell Activation via a PGE2-Dependent Mechanism, Stem Cells, 201634 2943-55.

\section{Figures}


A

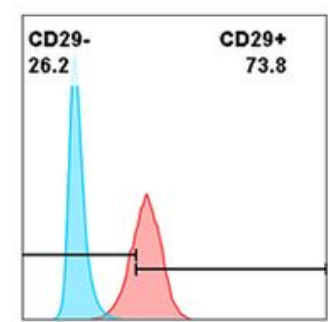

CD29

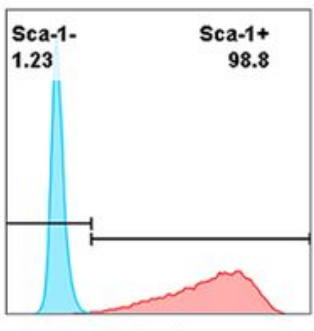

Sca-1

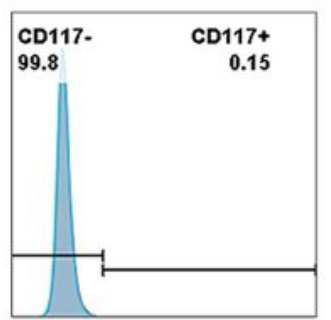

CD117

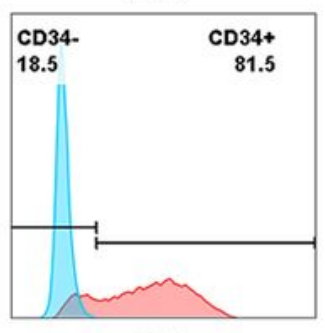

CD34

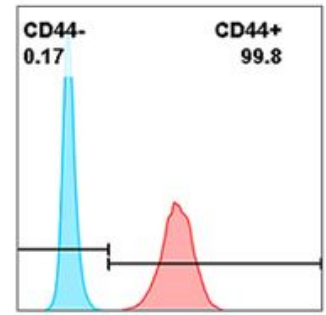

CD44

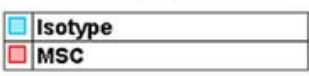

B

Experimental Protocol

Oh

$4 \mathrm{~h}$

48h

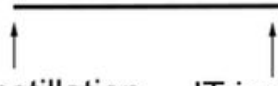

IT instillation IT instillation

Euthanasia

of LPS or PBS of MSCs or PBS

C
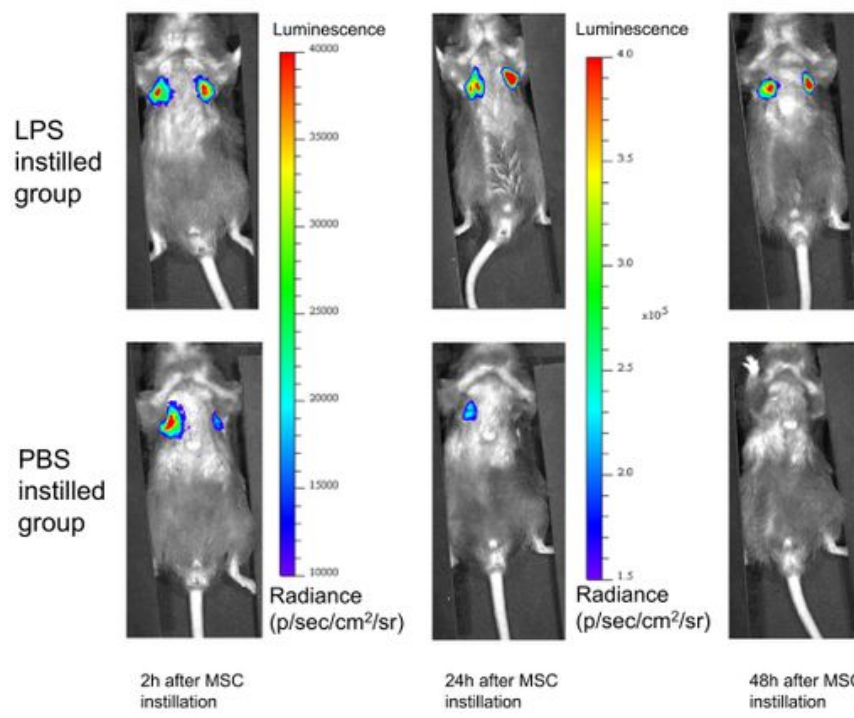

Luminescensce
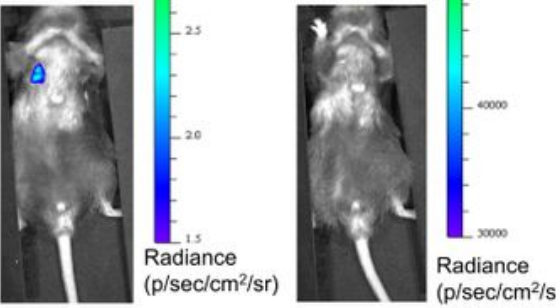

24h after MSC

48h after MSC instillation

\section{Figure 1}

MSC identification and in vivo location. (A) MSC identification by flow cytometry. (B) In vivo experimental protocol. PBS+PBS group: IT instillation of PBS at $0 \mathrm{~h}$ and PBS at $4 \mathrm{~h}$. LPS+PBS group: IT instillation of LPS at $0 \mathrm{~h}$ and PBS at $4 \mathrm{~h}$. LPS+MSC group: IT instillation of LPS at $0 \mathrm{~h}$ and MSCs at $4 \mathrm{~h}$. (C) Mouse in vivo imaging. Luc+ MSCs were detected in lungs. 
A
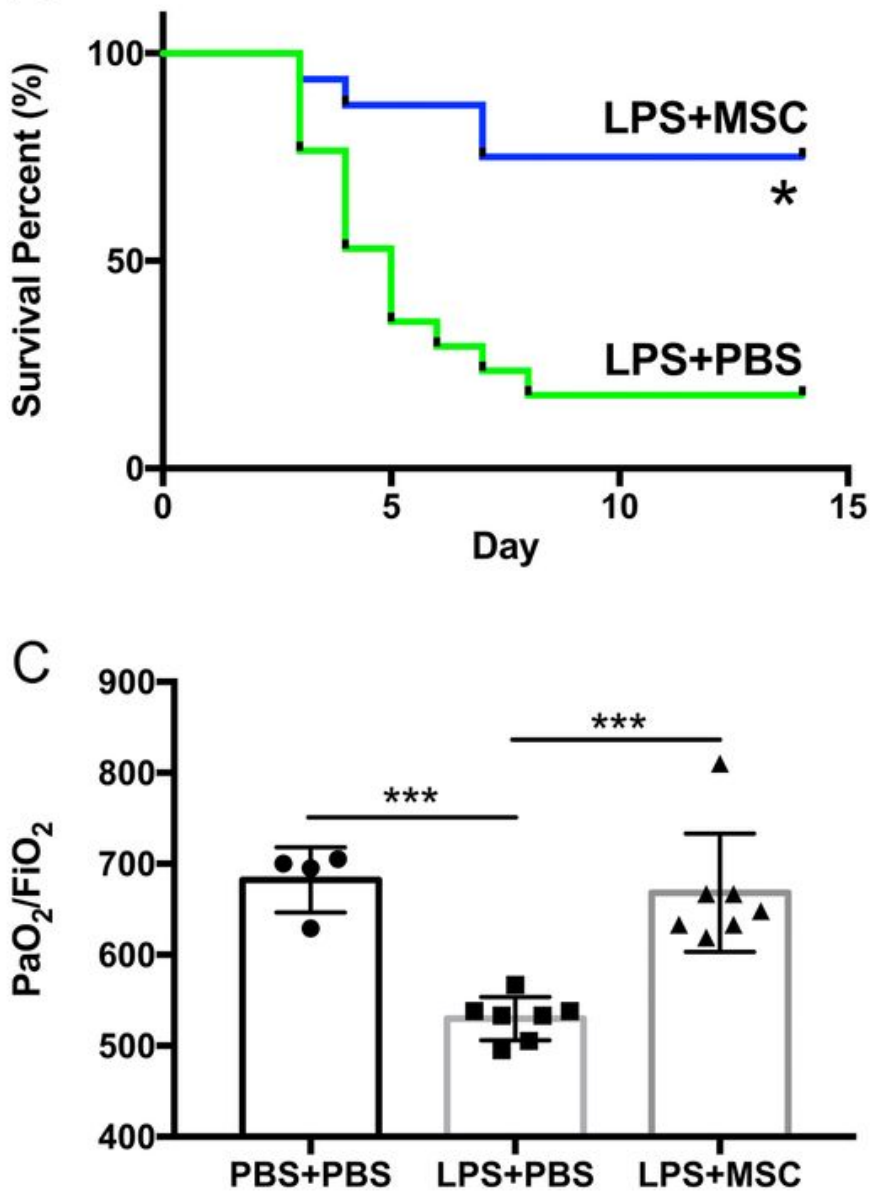

$E$

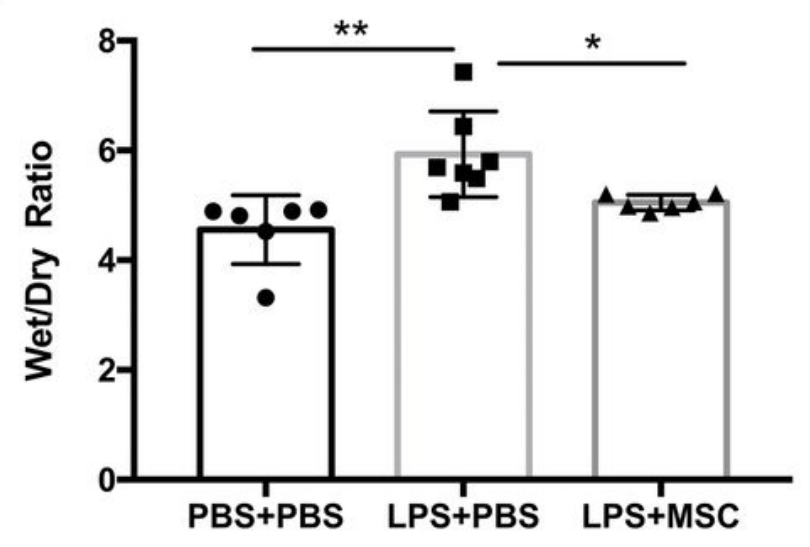

B
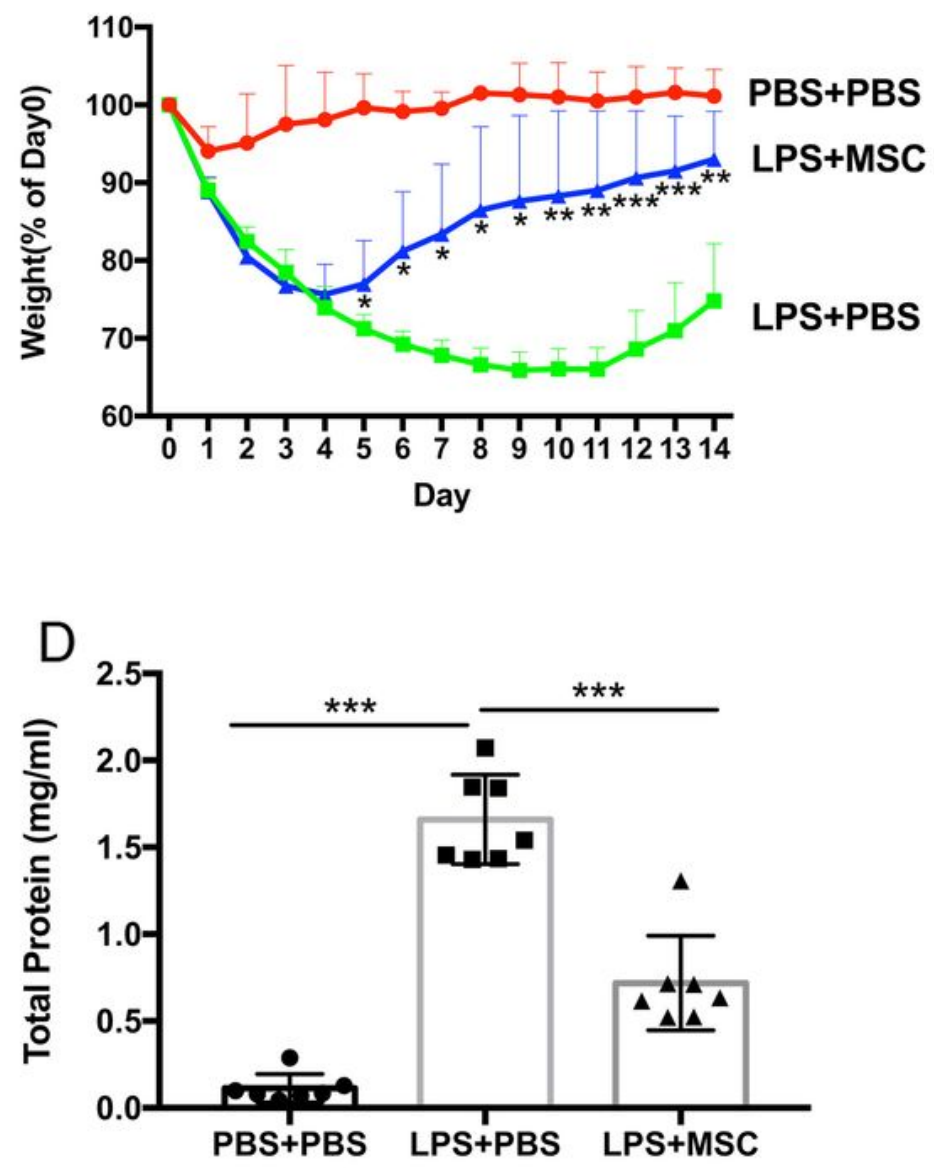

$\mathrm{F}$

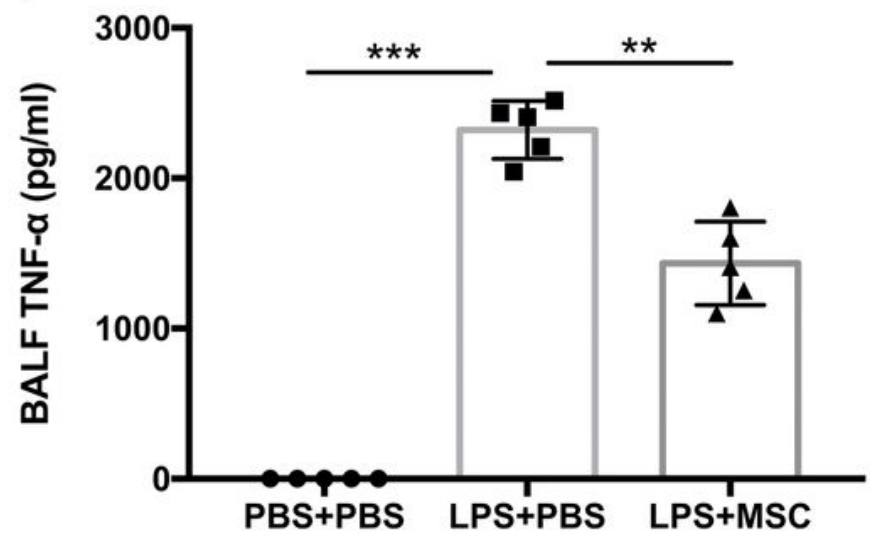

Figure 2

MSCs attenuated of ALI. (A) MSC administration significantly improved the 14-day survival rate of ALI mice ( $n=16-17$ per group). (B) Intratracheal administration of MSCs reduced the body weight decline of ALI mice from the 5th day ( $\mathrm{n}=3-6$ per group). (C) The P/F was improved by MSC treatment at $48 \mathrm{~h}$ after ALI instillation ( $n=4-8$ per group). (D) Intratracheal administration of MSCs significantly decreased total protein in BALF ( $n=7$ per group), (E) lung wet/dry ratio ( $n=6-7$ per group), and ( $F$ ) TNF-a concentration in 
BALF after ALI instillation compared with the findings in the LPS+PBS group ( $n=5$ per group). Data are presented as mean $\pm S D ; *, p<0.05 ; * *, p<0.01 ; * \star *, p<0.001$.

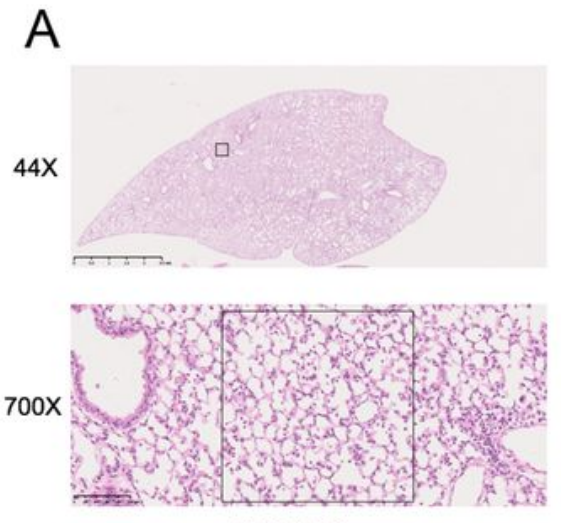

LPS+LPS
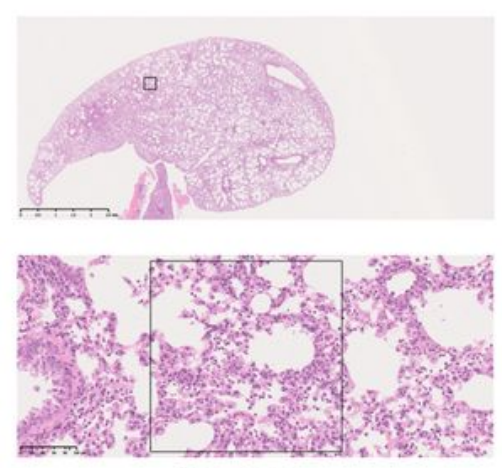

LPS+PBS
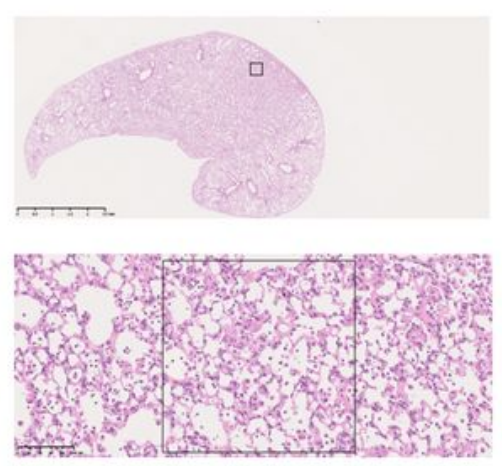

LPS+MSC

B
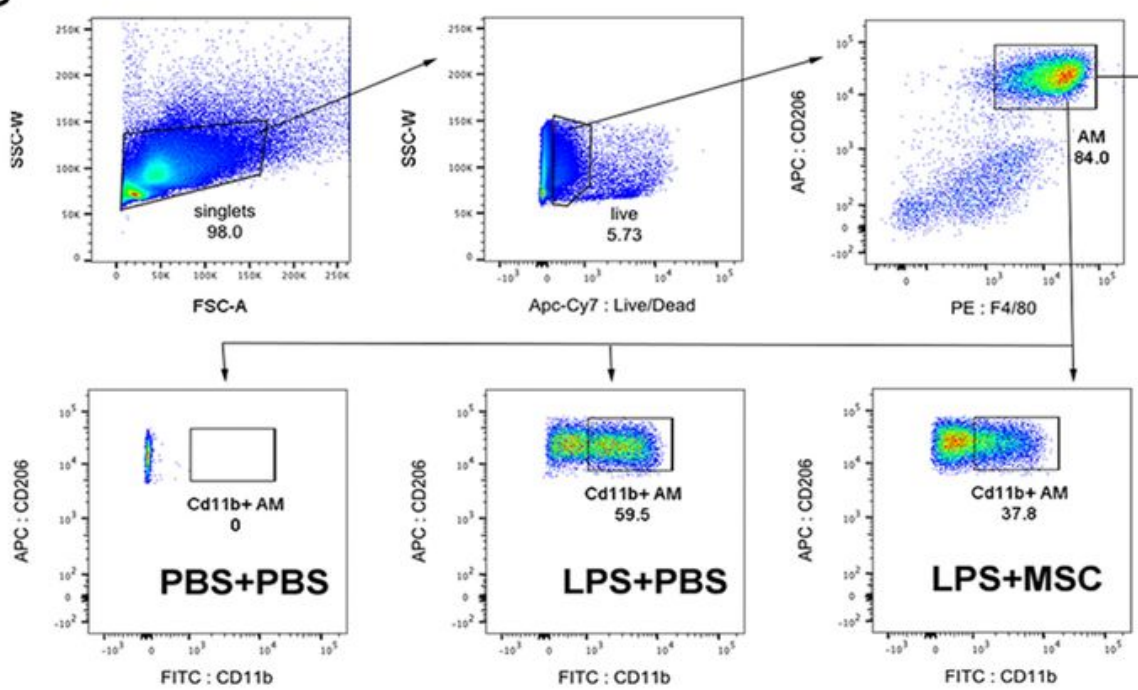

FITC : CD11b

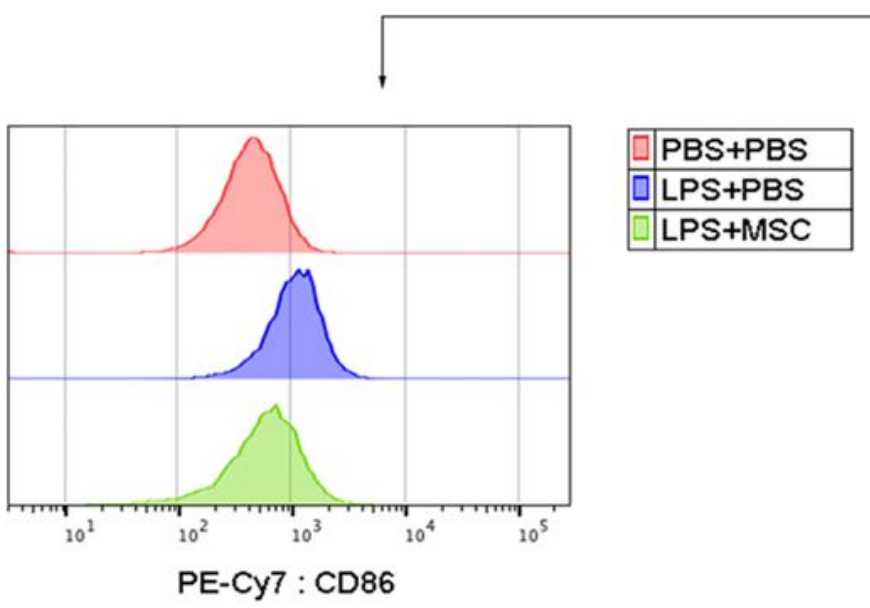

\section{Figure 3}

Effects of MSCs on pulmonary inflammation and alveolar macrophage activation. (A) MSCs improved lung injury as assessed by histology $48 \mathrm{~h}$ after $\mathrm{ALI}$ instillation. H\&E staining of lung sections in the LPS+PBS group showed that the alveolar epithelium was broken and thick without a complete alveolar 
profile, while MSC treatment protected the epithelium after LPS instillation. (B) The alveolar macrophages in BALF were defined as F4/80+ and CD206+. MSC treatment suppressed the expression of CD11b and CD86 on alveolar macrophages after ALI instillation. Data are presented as mean $\pm S D ; *, p<0.05 ; * \star$, $\mathrm{p}<0.01$.
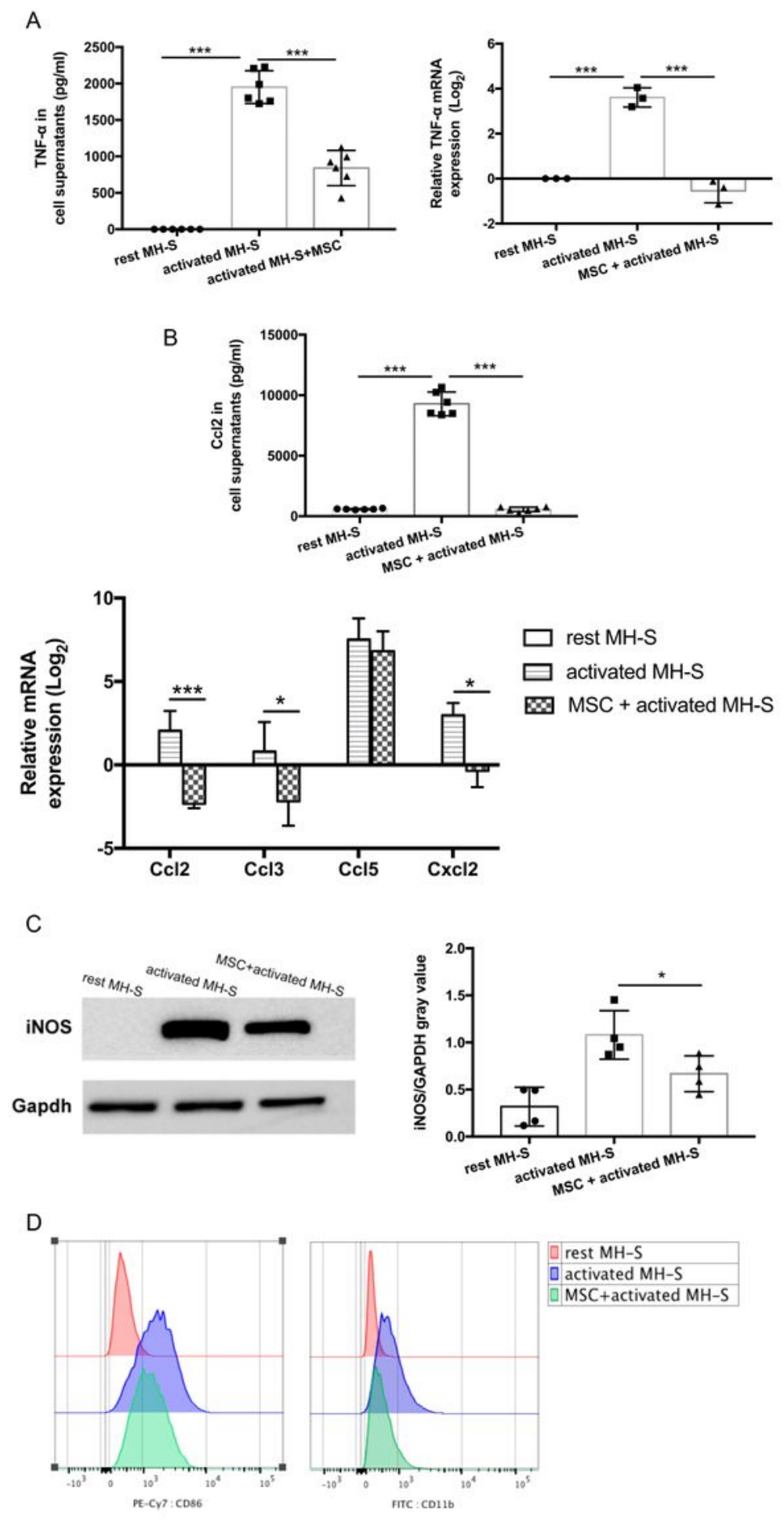

Figure 4 
MSCs inhibited MH-S cell activation. (A) TNF-a in supernatants and TNF-a mRNA of activated MH-S cells significantly decreased after co-culturing with MSCs for $24 \mathrm{~h}$ ( $\mathrm{n}=5$ per group). (B) The chemokine $\mathrm{Ccl} 2$ in supernatants and $\mathrm{Ccl} 2, \mathrm{Ccl} 3$, and $\mathrm{Cxcl} 2$ mRNA of activated MH-S cells co-culturing with MSCs declined after $24 \mathrm{~h}$ ( $\mathrm{n}=5$ per group). (C) Co-culturing with MSCs suppressed the iNOS protein synthesis of activated MH-S cells after $24 \mathrm{~h}$ ( $\mathrm{n}=5$ per group). (D) Activated MH-S cells expressed more CD $11 \mathrm{~b}$ and CD86 molecule than rest MH-S cells, but the expressions were suppressed by co-culturing with MSCs after $24 \mathrm{~h}$. Data are presented as mean $\pm \mathrm{SD} ;{ }^{*}, \mathrm{p}<0.05 ; * *, \mathrm{p}<0.01 ; * \star *, \mathrm{p}<0.001$.

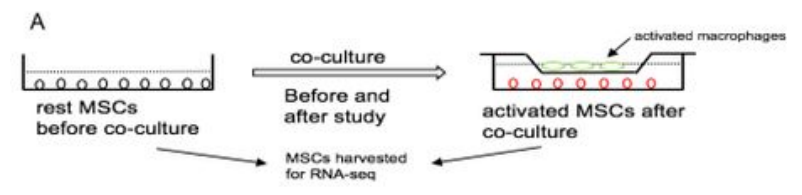

B
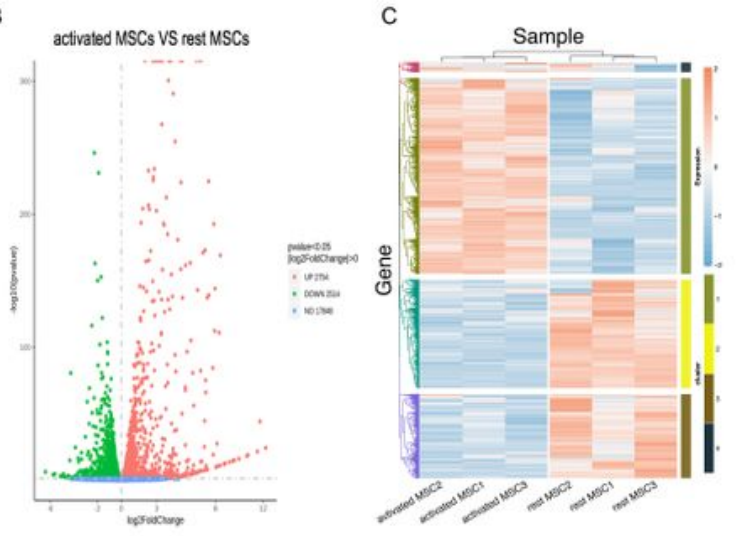

D
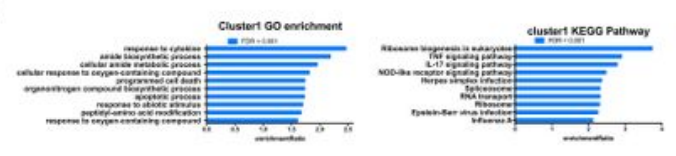

E

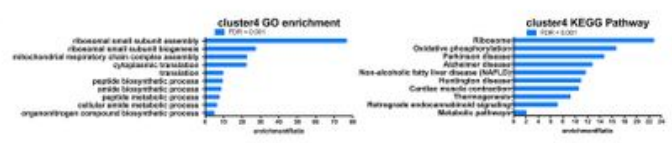

F

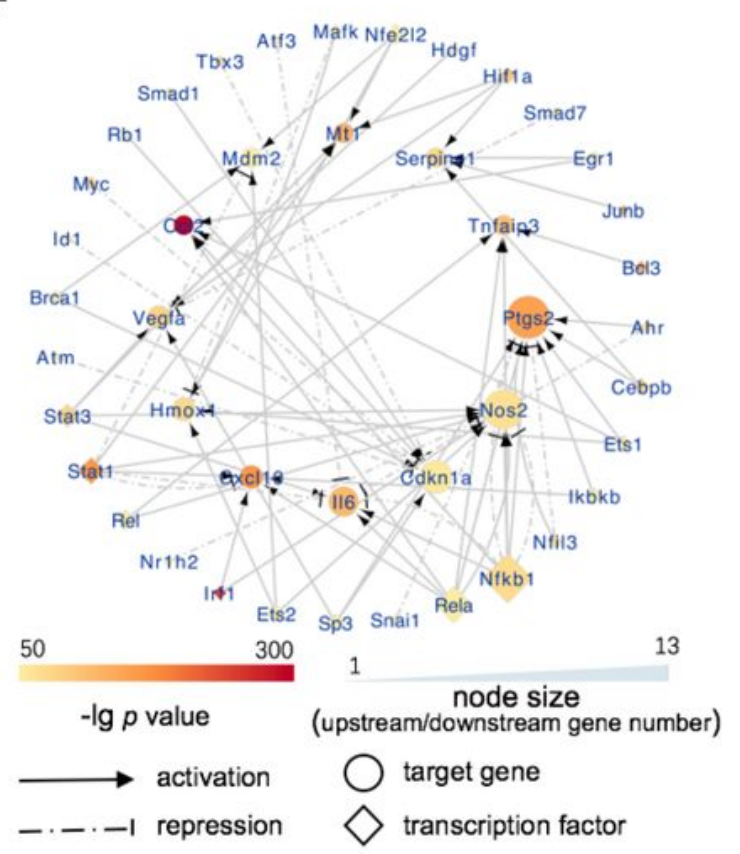




\section{Figure 5}

DEG profiling by RNA-seq. (A) RNA-seq protocol. (B) The upregulated and downregulated DEGs number of activated MSCs vs rest MSCs. (C) Heatmap of DEGs. (D) The GO enrichment and KEGG pathway analyses of cluster 1. (E) The GO enrichment and KEGG pathway analyses of cluster 4. (F) Transcription factor (TF)-target gene (TG) network. The nodes with different shapes represent TFs (rhombus) or TGs (circle), the color depth represents the gene expression level, the size of nodes represents regulated upstream number or regulating downstream number, the unbroken line and arrow represent activation, while the dotted line and segment represent repression. 
A

B
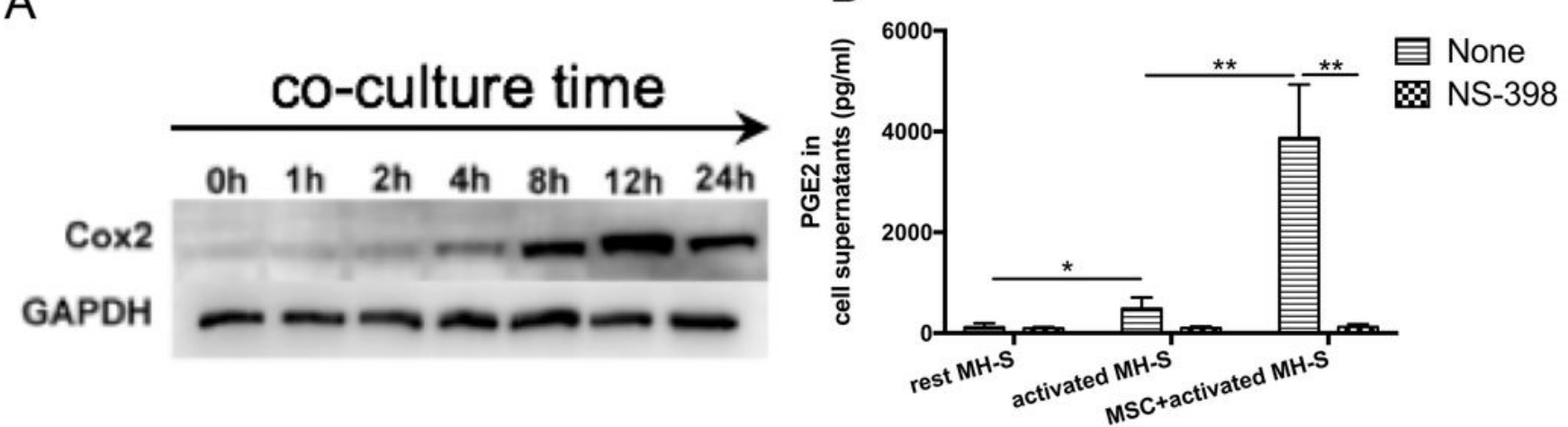

C

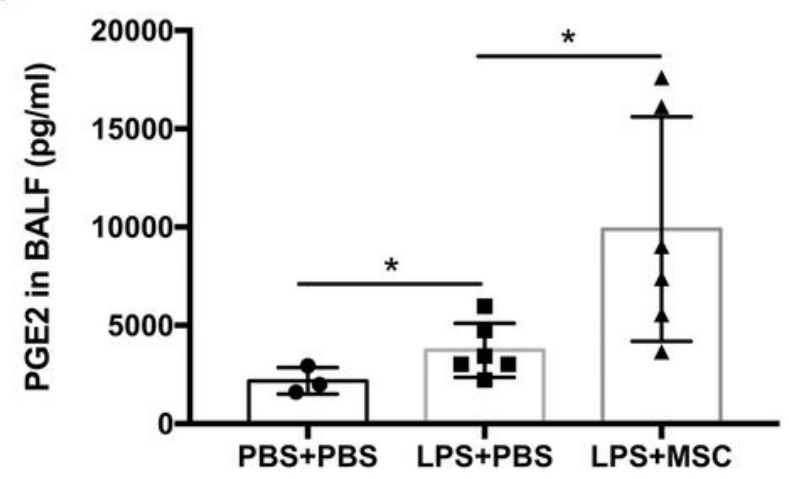

$\mathrm{D}$

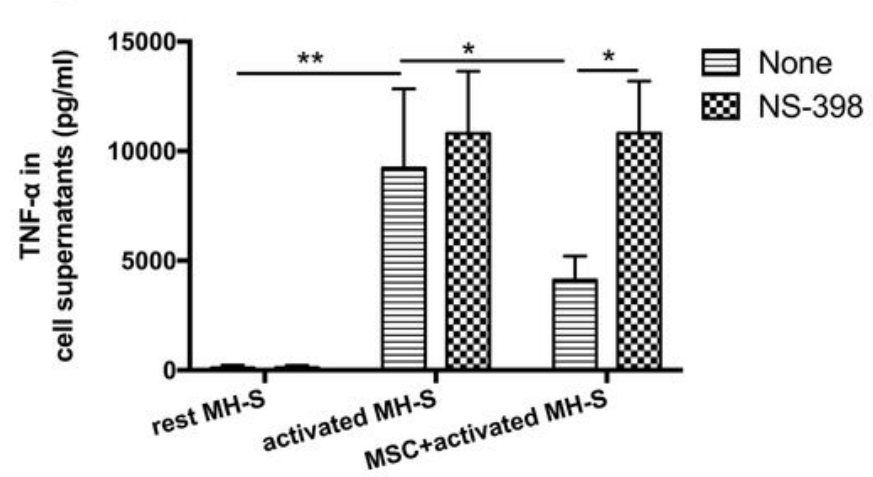

\section{Figure 6}

PGE2 secreted by MSCs contributed to the suppression of MH-S cell activation. (A) COX2 protein of MSCs was time-dependently induced. (B) The addition of MSCs dramatically increased the PGE2 level in supernatants after $24 \mathrm{~h}$ compared with that in the absence of MSCs and the COX2 inhibitor NS-398 (10 $\mu M$ ) significantly suppressed PGE2 secretion ( $n=5$ per group). (C) Intratracheal administration of MSCs increased the PGE2 level in BALF at $48 \mathrm{~h}$ after LPS instillation ( $\mathrm{n}=3-6$ per group). (D) Co-culturing with 
MSCs suppressed TNF-a secretion of activated MH-S cells, while the addition of NS-398 $(10 \mu \mathrm{M})$ reversed this suppression after 24 h. NS-398 had no effect on MH-S cells without MSCs ( $n=5$ per group). (E) Coculturing with MSCs suppressed CD86 expression of activated MH-S cells, while NS-398 $(10 \mu \mathrm{M})$ abolished this suppression after $24 \mathrm{~h}$. NS-398 had no effect on MH-S cells without MSCs. Data are presented as mean $\pm S D ; *, p<0.05 ; * \star, p<0.01 ; * \star \star, p<0.001$.
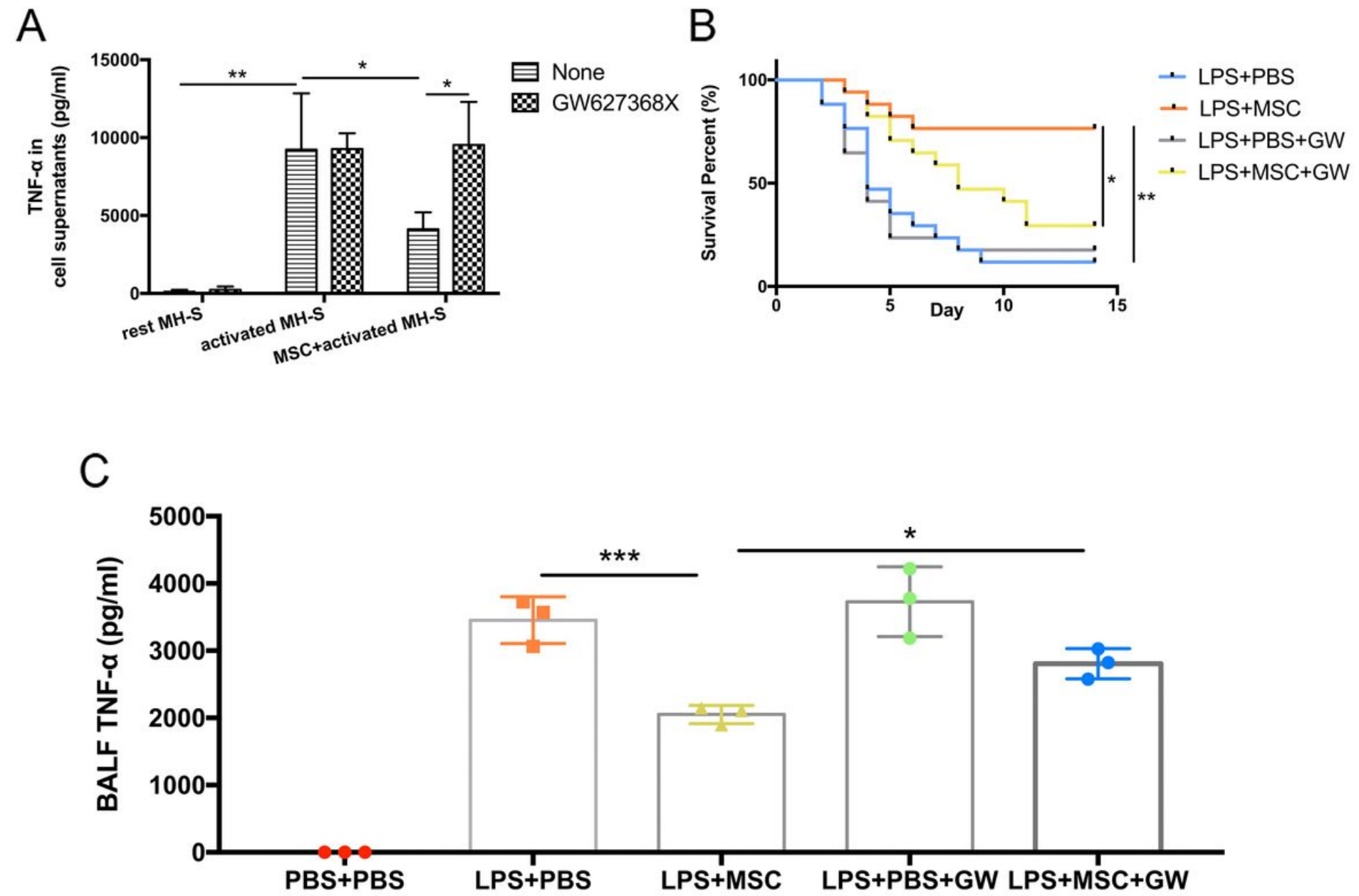

Figure 7

MSCs relieved ALI through secreting PGE2, which bound to EP4 receptor on alveolar macrophages. (A) Co-culturing with MSCs suppressed TNF-a secretion of MH-S cells, while the addition of the EP4 receptor antagonist GW627368X (10 $\mu \mathrm{M})$ reversed this suppression after $24 \mathrm{~h}$. GW627368X had no effect on $\mathrm{MH}$ $S$ cells without MSCs ( $n=5$ per group). (B) Intratracheal administration of MSCs significantly improved the 14-day survival rate of ALI mice compared with that in the LPS+PBS group. The addition of GW627368X significantly reversed this protective effect ( $n=16-17$ per group). (C) The addition of GW627368X significantly reversed the inhibitory effect of MSCs on TNF-a level in BALF at $48 \mathrm{~h}$ after LPS instillation $(n=3$ per group). Data are presented as mean $\pm S D ; *, p<0.05 ; * *, p<0.01 ; * \star *, p<0.001$. 


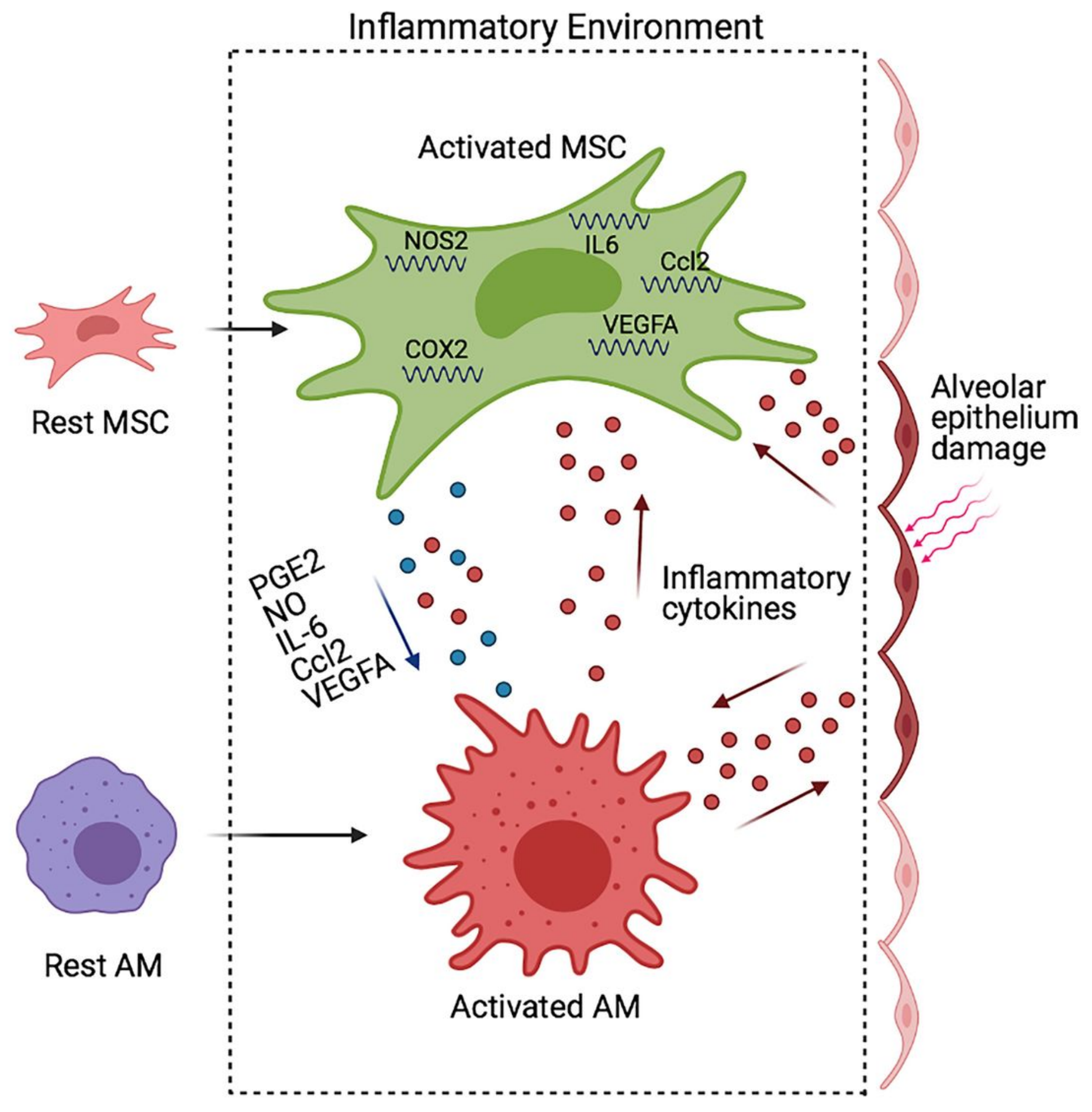

Figure 8

In ALI mouse model, the rest AMs were firstly activated by damaged tissues. The engrafting MSCs were subsequently activated by inflammatory cytokines from macrophages and secreted abundant immunosuppressive factors, like PGE2, NO. These immunosuppressive factors in turn suppressed macrophages activation and reduced further tissue damage. Activated MSCs could both exert anti- or proinflammatory capacity by releasing different cytokines which decided by inflammation degree and type. 


\section{Supplementary Files}

This is a list of supplementary files associated with this preprint. Click to download.

- SupplementalS1.xlsx 